\title{
Free vibration analysis of elastically supported Timoshenko columns with attached masses by transfer matrix and finite element methods
}

\author{
OKTAY DEMIRDAĞ \\ Pamukkale University, Engineering Faculty, Civil Engineering Department, \\ 20070 Kinikli Campus, Denizli, Turkey \\ e-mail: oktaydemirdag@pau.edu.tr
}

MS received 10 December 2007; accepted 15 January 2008

\begin{abstract}
This paper deals with the free vibration of Timoshenko columns with attached masses having rotary inertia. The support of the model is elastically restrained against rotation. The concept of fixity factor is used to define the stiffness of the elastic connection relative to that of the column. The governing equation of the column elements is solved by applying the separation of variables method in the transfer matrix method (TMM) algorithm. The same problems are solved, also, by finite element method (FEM) algorithm in which the matrices in equation of motion are obtained for Timoshenko column, and the results are compared with the ones of TMM. The comparison graphs are presented in numerical analysis to show the effectiveness of the considered methods, and it is resulted that FEM gives closer results to TMM.
\end{abstract}

Keywords. Free vibration; elastic support; Timoshenko column; transfer matrix; finite element.

\section{Introduction}

Bapat and Bapat investigated the natural frequencies of an Euler beam with concentrated masses, and modelled all supports by elastic springs against rotation and translation (Bapat \& Bapat 1987). Karami et al (2003) proposed a differential quadrature element method for free vibration analysis of non-uniform Timoshenko beams with elastic support and attachments. Lin and Chang (2005) studied free vibration analysis of multi-span Timoshenko beam with an arbitrary number of flexible constraints by TMM. Posiadala (1997) considered the transverse free vibration of Timoshenko beams having rotation and translation springs, concentrated mass with moment of inertia, linear undamped oscillators and additional supports, and obtained the frequency equation by Lagrange multiplier formalism. TMM is used with Holzer method for torsional vibration of systems with concentrated masses (Hurty \& Rubinstein 1964), and with Myklestad-Thomson method for flexural vibrations of discrete systems with concentrated masses (Thomson 1981). Esmailzadeh and Ohadi (2000) made vibration and stability analysis of non-uniform Timoshenko beams under axial and distributed tangential loads. 


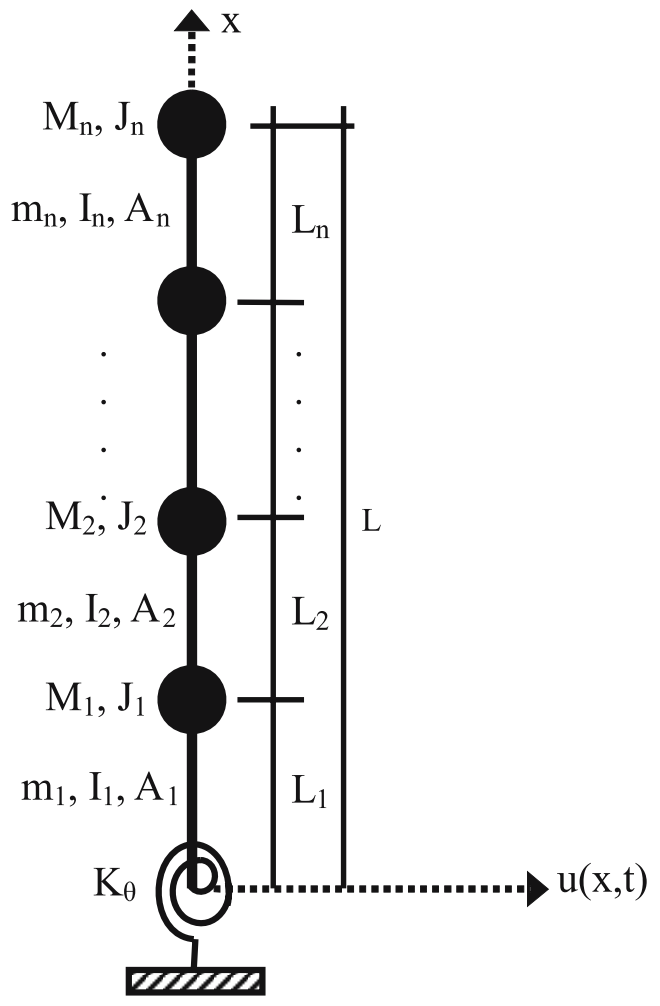

Figure 1. Model of an elastically-supported Timoshenko column with attached masses.

Gökdağ and Kopmaz (2005) studied the coupled flexural-torsional free and forced vibrations of a Timoshenko beam with tip and/or in-span attachments. Özkaya (2002) obtained the nonlinear equations of motion for transverse vibrations of a simply supported beam carrying concentrated masses. Wu and Chiang (2004) studied about natural frequencies and mode shapes of the double-tapered wedge beams carrying multiple point masses. Wu (2006) investigated free vibration characteristics of a beam carrying multiple two-dof spring-mass systems. Wu and Chen (2001) studied free vibration analysis of a Timoshenko beam carrying multiple spring-mass systems by using the numerical assembly technique. Rao et al (2006) examined large amplitude free vibration behaviour of uniform Timoshenko beams with central point concentrated masses. Ruta applied Chebyshev series approximation to solve vibration problem of non-prismatic Timoshenko beam resting a two-parameter elastic foundation (Ruta 2006). Lee et al (2004) formulated spectral element model for axially moving Timoshenko beam under a uniform axial tension. Salarieh and Ghorashi (2006) analysed free vibration of a cantilever Timoshenko beam with a rigid tip mass. Ferreira and Fasshuer (2006) presented a study of free vibration of Timoshenko beams and Mindlin plates by RBF-pseudospectral method.

The mathematical model of $n$ uniform Timoshenko columns with $n$ attached masses given in figure 1 is used in this study for multistory frames. Elastic support is modelled by rotation spring. In order to reflect the relative stiffness of the column and the rotational spring, an end fixity factor is defined. Thus, the fixity factor is defined in (1) from the rotational stiffness 
so that it takes as limits: null (0) value for a theoretically pinned joint and unity (1) for a theoretically rigid one (Cabrero \& Bayo 2005).

$$
f=\frac{1}{1+\frac{3 E I}{K_{\theta} L}} \quad(0 \leq f \leq 1)
$$

where $E I$ and $L$ are flexural rigidity and length of the Timoshenko column, $K_{\theta}$ is the rotational spring constant. The governing equation of free vibration is derived by including bending and shear deformations with rotary inertia of the columns. The rotary inertia of the attached masses is also included in the analysis. A TMM approach considering the continuity relations of displacement, slope, moment and shear at the interface of adjacent columns is performed to determine eigenfrequencies of the model. Considering the compatibility conditions at the interface of adjacent columns the relations between two adjacent spans is obtained; thus, exact values of eigenfrequencies of the entire system are determined for different number of masses by using TMM algorithm. Another method, finite element method (FEM), is also used to obtain the frequencies of the model. Stiffness and mass matrices of the Timoshenko column are obtained to solve the matrix equation of motion; thus, the eigenvalues being the frequencies are calculated using FEM algorithm.

\section{Analysis by TMM}

\subsection{Determining eigenfunction}

Differential equation of motion for the $i$ th Timoshenko column is

$$
\frac{\partial^{4} u_{i}}{\partial x_{i}^{4}}-\left(\frac{m_{i} k_{i}}{A G_{i}}+\frac{m_{i} r_{i}^{2}}{E I_{i}}\right) \frac{\partial^{4} u_{i}}{\partial x_{i}^{2} \partial t^{2}}+\frac{m_{i}^{2} r_{i}^{2} k_{i}}{E I_{i} A G_{i}} \frac{\partial^{4} u_{i}}{\partial t^{4}}+\frac{m_{i}}{E I_{i}} \frac{\partial^{2} u_{i}}{\partial t^{2}}=0,
$$

where $u_{i}\left(x_{i}, t\right), m_{i}, r_{i}, k_{i}, E I_{i}$ and $A G_{i}$, are displacement at $x_{i}\left(0 \leq x_{i} \leq L_{i}\right)$, distributed mass, radius of gyration, effective shear area factor due to cross-section geometry, flexural and shear rigidities, respectively, of the $i$ th column. Applying the separation of variables method to (2) in the form of (3) for $T(t) \neq 0$ and re-arranging with the dimensionless parameters gives the eigenfunction $X\left(x_{i}\right)$ of the $i$ th storey column as in (4).

$$
\begin{aligned}
u_{i}\left(x_{i}, t\right) & =X_{i}\left(x_{i}\right) T(t)=X_{i}\left(x_{i}\right)[A \sin (\omega t)+B \cos (\omega t)] \\
X_{i}\left(x_{i}\right) & =C_{1 i} \sinh \left(\lambda_{1 i} x_{i}\right)+C_{2 i} \cosh \left(\lambda_{1 i} x_{i}\right)+C_{3 i} \sin \left(\lambda_{2 i} x_{i}\right)+C_{4 i} \cos \left(\lambda_{2 i} x_{i}\right),
\end{aligned}
$$

where $\alpha_{1 i}=m_{i} k_{i} \omega^{2} / A G_{i} ; \alpha_{2 i}=m_{i} \omega^{2} / E I_{i} ; \alpha_{3 i}=\alpha_{1 i}+\alpha_{2 i} r_{i}^{2} ; \Delta_{i}=\left(\alpha_{1 i}-\alpha_{2 i} r_{i}^{2}\right)^{2}+4 \alpha_{2 i}$; $n_{1 i}=\left(-\alpha_{3 i}+\sqrt{\Delta_{i}}\right) / 2 ; n_{2 i}=\left(-\alpha_{3 i}-\sqrt{\Delta_{i}}\right) / 2 ; \lambda_{1 i}=\sqrt{n_{1 i}} ; \lambda_{2 i}=\sqrt{\left|n_{2 i}\right|} ; C_{1 i} \ldots C_{4 i}$ are integration constants. Moment, shear, slope functions of the $i$ th Timoshenko column are as follows (Tuma \& Cheng 1983).

$$
\begin{aligned}
M_{i}\left(x_{i}, t\right) & =-E I_{i} u_{i}^{\prime \prime}\left(x_{i}, t\right)-E I_{i} \alpha_{1} u_{i}\left(x_{i}, t\right) \\
V_{i}\left(x_{i}, t\right) & =\left[-E I_{i} /\left(1-\alpha_{1 i} r_{i}^{2}\right)\right]\left[u_{i}^{\prime \prime \prime}\left(x_{i}, t\right)+\alpha_{3 i} u_{i}^{\prime}\left(x_{i}, t\right)\right] \\
\theta_{i}\left(x_{i}, t\right) & =u_{i}^{\prime}\left(x_{i}, t\right)-V_{i}\left(x_{i}, t\right) k_{i} / A G_{i}
\end{aligned}
$$




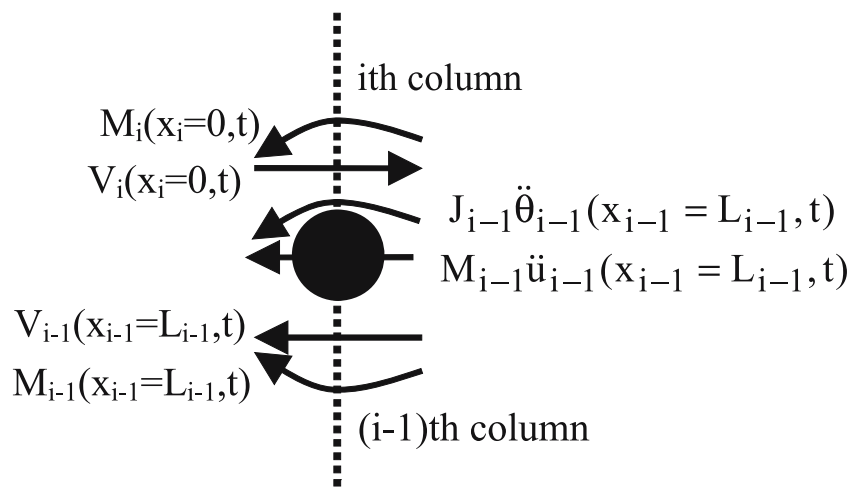

Figure 2. Free body diagram for the interface of $(i-1)$ th and $i$ th columns.

\subsection{Boundary conditions}

Boundary conditions at the interface of the adjacent $(i-1)$ th and $i$ th columns (figure 2) are written as in (6) using the continuity of displacement and slope and the equilibrium of moment and shear (Demirdağ 2005).

$$
\begin{aligned}
& u_{i-1}\left(x_{i-1}=L_{i-1}, t\right)=u_{i}\left(x_{i}=0, t\right) \\
& \theta_{i-1}\left(x_{i-1}=L_{i-1}, t\right)=\theta_{i}\left(x_{i}=0, t\right) \\
& M_{i-1}\left(x_{i-1}=L_{i-1}, t\right)-J_{i-1} \ddot{\theta}_{i-1}\left(x_{i-1}=L_{i-1}, t\right)=M_{i}\left(x_{i}=0, t\right) \\
& V_{i-1}\left(x_{i-1}=L_{i-1}, t\right)+M_{i-1} \ddot{u}_{i-1}\left(x_{i-1}=L_{i-1}, t\right)=V_{i}\left(x_{i}=0, t\right),
\end{aligned}
$$

where $M_{i}$ and $J_{i}$ are $i$ th attached mass and its rotary inertia, $L_{i}$ is length of the $i$ th column. Since continuity of displacement and slope is not valid for the support and the $n$th attached mass, one gets $4(n-1)$ relations from (6). However, four more relations are needed for the entire system, two given in (7) from the elastic support in figure 3 and two given in (8) from the $n$th attached mass in figure 4 where $K_{\theta}$ is rotational spring constant (Demirdağ 2005).

$$
\begin{aligned}
& u_{1}\left(x_{1}=0, t\right)=0 \\
& \left.M_{1}\left(x_{1}=0, t\right)=-K_{\theta} \theta_{1}\left(x_{1}=0, t\right)\right\}
\end{aligned}
$$

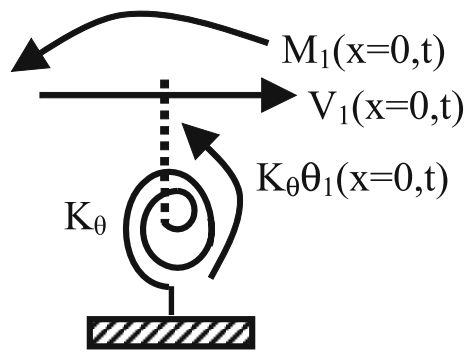

Figure 3. Free body diagram of elastic support. 


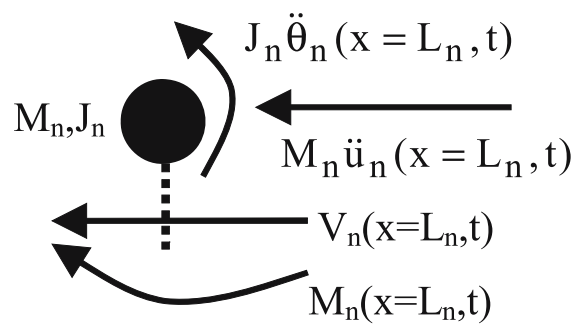

Figure 4. Free body diagram of $n$th mass.

$$
\left.\begin{array}{l}
M_{n}\left(x_{n}=L_{n}, t\right)=J_{n} \ddot{\theta}_{n}\left(x_{n}=L_{n}, t\right) \\
V_{n}\left(x_{n}=L_{n}, t\right)=-M_{n} \ddot{u}_{n}\left(x_{n}=L_{n}, t\right)
\end{array}\right\}
$$

\subsection{Obtaining transfer matrix}

The relation between $C_{1 i} \ldots C_{4 i}$ and $C_{1 i-1} \ldots C_{4 i-1}$ is written from (6) in matrix form as

$$
\left\{\begin{array}{l}
C_{1 i} \\
C_{2 i} \\
C_{3 i} \\
C_{4 i}
\end{array}\right\}=\left[T_{i}\right]\left\{\begin{array}{l}
C_{1 i-1} \\
C_{2 i-1} \\
C_{3 i-1} \\
C_{4 i-1}
\end{array}\right\}=\left[\begin{array}{llll}
T_{i 11} & T_{i 12} & T_{i 13} & T_{i 14} \\
T_{i 21} & T_{i 22} & T_{i 23} & T_{i 24} \\
T_{i 31} & T_{i 32} & T_{i 33} & T_{i 34} \\
T_{i 41} & T_{i 42} & T_{i 43} & T_{i 44}
\end{array}\right]\left\{\begin{array}{l}
C_{1 i-1} \\
C_{2 i-1} \\
C_{3 i-1} \\
C_{4 i-1}
\end{array}\right\}(i=2,3, \ldots, n)
$$

where

$$
\begin{aligned}
& T_{i 11}=\alpha_{26 i} \alpha_{9 i-1} c h_{i-1}+\alpha_{27 i} \alpha_{14 i-1} ; \quad T_{i 12}=\alpha_{26 i} \alpha_{9 i-1} s h_{i-1}+\alpha_{27 i} \alpha_{15 i-1} \\
& T_{i 13}=\alpha_{26 i} \alpha_{10 i-1} c_{i-1}+\alpha_{27 i} \alpha_{16 i-1} ; \quad T_{i 14}=-\alpha_{26 i} \alpha_{10 i-1} s_{i-1}+\alpha_{27 i} \alpha_{17 i-1} \\
& T_{i 21}=\alpha_{31 i} s h_{i-1}-\alpha_{30 i} \alpha_{21 i-1} ; \quad T_{i 22}=\alpha_{31 i} c h_{i-1}-\alpha_{30 i} \alpha_{22 i-1} \\
& T_{i 23}=\alpha_{31 i} s_{i-1}-\alpha_{30 i} \alpha_{23 i-1} ; \quad T_{i 24}=\alpha_{31 i} c_{i-1}-\alpha_{30 i} \alpha_{24 i-1} ; \\
& T_{i 31}=\alpha_{28 i} \alpha_{9 i-1} c h_{i-1}-\alpha_{29 i} \alpha_{14 i-1} ; \quad T_{i 32}=\alpha_{28 i} \alpha_{9 i-1} s h_{i-1}-\alpha_{29 i} \alpha_{15 i-1} \\
& T_{i 33}=\alpha_{28 i} \alpha_{10 i-1} c_{i-1}-\alpha_{29 i} \alpha_{16 i-1} ; \quad T_{i 34}=-\alpha_{28 i} \alpha_{10 i-1} s_{i-1}-\alpha_{29 i} \alpha_{17 i-1} \\
& T_{i 41}=\alpha_{32 i} s h_{i-1}+\alpha_{30 i} \alpha_{21 i-1} ; \quad T_{i 42}=\alpha_{32 i} c h_{i-1}+\alpha_{30 i} \alpha_{22 i-1} \\
& T_{i 43}=\alpha_{32 i} s_{i-1}+\alpha_{30 i} \alpha_{23 i-1} ; \quad T_{i 44}=\alpha_{32 i} c_{i-1}+\alpha_{30 i} \alpha_{24 i-1} ; \\
& \alpha_{32 i}=\alpha_{4 i} \alpha_{30 i} ; \quad \alpha_{31 i}=\alpha_{5 i} \alpha_{30 i} ; \quad \alpha_{30 i}=1 /\left(\alpha_{4 i}+\alpha_{5 i}\right) \\
& \alpha_{28 i}=\alpha_{12 i} / \alpha_{25 i} ; \quad \alpha_{27 i}=\alpha_{10 i} / \alpha_{25 i} ; \quad \alpha_{26 i}=\alpha_{13 i} / \alpha_{25 i} ; \\
& \alpha_{25 i}=\alpha_{9 i} \alpha_{13 i}+\alpha_{12 i} \alpha_{10 i} ; \quad \alpha_{24 i}=\alpha_{5 i} c_{i}-\alpha_{20 i} s_{i} ; \\
& \alpha_{23 i}=\alpha_{5 i} s_{i}+\alpha_{20 i} c_{i} ; \quad \alpha_{22 i}=\alpha_{19 i} s h_{i}-\alpha_{4 i} c h_{i} ; \\
& \alpha_{21 i}=\alpha_{19 i} c h_{i}-\alpha_{4 i} s h_{i} ; \quad \alpha_{20 i}=\alpha_{18 i} \alpha_{10 i} ; \quad \alpha_{19 i}=\alpha_{18 i} \alpha_{9 i}
\end{aligned}
$$




$$
\begin{aligned}
& \alpha_{18 i}=J_{i} \omega^{2} ; \quad \alpha_{17 i}=\alpha_{13 i} s_{i}-\alpha_{11 i} c_{i} ; \quad \alpha_{16 i}=-\alpha_{13 i} c_{i}-\alpha_{11 i} s_{i} ; \\
& \alpha_{15 i}=\alpha_{12 i} s h_{i}-\alpha_{11 i} c h_{i} ; \quad \alpha_{14 i}=\alpha_{12 i} c h_{i}-\alpha_{11 i} s h_{i} ; \quad \alpha_{13 i}=\alpha_{6 i} \alpha_{8 i} ; \\
& \alpha_{12 i}=\alpha_{6 i} \alpha_{8 i} ; \quad \alpha_{11 i}=M_{i} \omega^{2} ; \quad \alpha_{10 i}=\lambda_{2 i}+\alpha_{6 i} \alpha_{8 i} k_{i} / A G_{i} ; \\
& \alpha_{9 i}=\lambda_{1 i}-\alpha_{6 i} \alpha_{7 i} k_{i} / A G_{i} ; \quad \alpha_{8 i}=\lambda_{2 i}\left(\lambda_{2 i}^{2}-\alpha_{3 i}\right) ; \quad \alpha_{7 i}=\lambda_{1 i}\left(\lambda_{1 i}^{2}+\alpha_{3 i}\right) ; \\
& \alpha_{6 i}=-E I_{i} /\left(1-\alpha_{1 i} r_{i}^{2}\right) ; \quad \alpha_{5 i}=E I_{i}\left(\lambda_{2 i}^{2}-\alpha_{1 i}\right) ; \\
& \alpha_{4 i}=E I_{i}\left(\lambda_{1 i}^{2}+\alpha_{1 i}\right) \quad s h_{i}=\sinh \left(\lambda_{1 i} L_{i}\right) ; \quad c h_{i}=\cosh \left(\lambda_{1 i} L_{i}\right) ; \\
& s_{i}=\sin \left(\lambda_{2 i} L_{i}\right) ; \quad c_{i}=\cos \left(\lambda_{2 i} L_{i}\right) .
\end{aligned}
$$

Applying (9) consecutively for $n$ storey gives

$$
\left\{\begin{array}{l}
C_{1 n} \\
C_{2 n} \\
C_{3 n} \\
C_{4 n}
\end{array}\right\}=\left[T_{t}\right]\left\{\begin{array}{l}
C_{11} \\
C_{21} \\
C_{31} \\
C_{41}
\end{array}\right\}=\left[T_{n}\right]\left[T_{n-1}\right] \ldots\left[T_{3}\right]\left[T_{2}\right]\left\{\begin{array}{l}
C_{11} \\
C_{21} \\
C_{31} \\
C_{41}
\end{array}\right\},
$$

where $\left[T_{t}\right]$ is the transfer matrix of the entire system. Substituting (10) into (8) gives two more equation related to $C_{11} \ldots C_{41}$, therefore, there exists 4 homogeneous equations together with (7) that characterize free vibration of the entire system as

$$
[F]\left\{\begin{array}{l}
C_{11} \\
C_{21} \\
C_{31} \\
C_{41}
\end{array}\right\}=\left\{\begin{array}{l}
0 \\
0 \\
0 \\
0
\end{array}\right\},
$$

where $[F]$ is coefficient matrix. Equating the determinant of $[F]$ to zero gives frequency equation of the entire system, and every root of this frequency equation is the eigenfrequency of the model. These frequencies are computed by a program written by the authors considering the secant method (Low 1991).

\section{Analysis by FEM}

\subsection{Element matrices}

Prezemieniecki (1968) obtained the stiffness and mass matrices for two-dimensional problems of a uniform Timoshenko beam-column including shear deformation and rotary inertia effects together with bending deformation, respectively as

$$
\left[K_{e}\right]=\frac{E I}{L^{3}(1+\phi)}\left[\begin{array}{cccc}
12 & 6 L & -12 & 6 L \\
6 L & 4 L^{2}+\phi L^{2} & -6 L & 2 L^{2}-\phi L^{2} \\
-12 & -6 L & 12 & -6 L \\
6 L & 2 L^{2}-\phi L^{2} & -6 L & 4 L^{2}+\phi L^{2}
\end{array}\right]
$$




$$
\left[M_{e}\right]=\frac{m L}{(1+\phi)^{2}}\left[\begin{array}{cccc}
m_{1}+\frac{r^{2}}{L^{2}} m_{7} & m_{2}+\frac{r^{2}}{L^{2}} m_{8} & m_{3}-\frac{r^{2}}{L^{2}} m_{7} & -m_{4}+\frac{r^{2}}{L^{2}} m_{8} \\
m_{2}+\frac{r^{2}}{L^{2}} m_{8} & m_{5}+\frac{r^{2}}{L^{2}} m_{9} & m_{4}-\frac{r^{2}}{L^{2}} m_{8} & -m_{6}+\frac{r^{2}}{L^{2}} m_{10} \\
m_{3}-\frac{r^{2}}{L^{2}} m_{7} & m_{4}-\frac{r^{2}}{L^{2}} m_{8} & m_{1}+\frac{r^{2}}{L^{2}} m_{7} & -m_{2}-\frac{r^{2}}{L^{2}} m_{8} \\
-m_{4}+\frac{r^{2}}{L^{2}} m_{8} & -m_{6}+\frac{r^{2}}{L^{2}} m_{10} & -m_{2}-\frac{r^{2}}{L^{2}} m_{8} & m_{5}+\frac{r^{2}}{L^{2}} m_{9}
\end{array}\right]
$$

where $\phi=12 E I / k A G L^{2}, r^{2}=I / A$,

$$
\begin{aligned}
& m_{1}=\frac{13}{35}+\frac{7 \phi}{10}+\frac{\phi^{2}}{3}, \quad m_{2}=\frac{11 L}{210}+\left(\frac{11 \phi}{120}+\frac{\phi^{2}}{24}\right) L, \\
& m_{3}=\frac{9}{70}+\frac{3 \phi}{10}+\frac{\phi^{2}}{6}, \quad m_{4}=\frac{13 L}{420}+\left(\frac{3 \phi}{40}+\frac{\phi^{2}}{24}\right) L, \\
& m_{5}=\frac{L^{2}}{105}+\left(\frac{\phi}{60}+\frac{\phi^{2}}{120}\right) L^{2}, \quad m_{6}=\frac{L^{2}}{140}+\left(\frac{\phi}{60}+\frac{\phi^{2}}{120}\right) L^{2}, \\
& m_{7}=\frac{6}{5}, \quad m_{8}=\left(\frac{1}{10}-\frac{\phi}{2}\right) L, \quad m_{9}=\left(\frac{2}{15}+\frac{\phi}{6}+\frac{\phi^{2}}{3}\right) L^{2}, \\
& m_{10}=\left(\frac{-1}{30}-\frac{\phi}{6}+\frac{\phi^{2}}{6}\right) L^{2},
\end{aligned}
$$

and $E I, k, A G, L$ are the parameters defined for the $i$ th column above. It is clear that if the shear deformations and rotatory inertia effects are neglected that is, $\phi=0$ and $r=0$, (12) and (13) will be the stiffness and mass matrices of a Bernoulli-Euler model.

The stiffness and mass matrices are obtained for each element using (12) and (13), and they are added appropriately to obtain the system stiffness $([K])$ and mass $([M])$ matrices by a method of assembling the system stiffness and mass matrices called the direct method (Paz 1997). In addition, the concentrated masses $\left(M_{i}\right)$ and their rotary inertia $\left(J_{i}\right)$ values are added in the system mass matrix directly to the mass coefficient $m_{i i}$, and the rotational spring constant value is added in the system stiffness matrix to the stiffness coefficient $k_{22}$. The equations of motion as functions of the nodal coordinates may then be established by imposing conditions of dynamic equilibrium between the inertial $\left[F_{I}(t)\right]$ and elastic $\left[F_{S}(t)\right]$ forces, that is,

$$
\left\{F_{I}(t)\right\}+\left\{F_{S}(t)\right\}=[M]\{\ddot{u}\}+[K]\{u\}=0 .
$$

If it assumed that $u_{i}(t)=a_{i} \sin (\omega t), u_{i}$ and $a_{i}$ being the displacement and the amplitude at the $i$ th concentrated mass, then (14) becomes an eigenvalue problem as

$$
\left[[D]-\frac{1}{\omega^{2}}[I]\right]\{a\}=\{0\} \rightarrow\left|[D]-\frac{1}{\omega^{2}}[I]\right|=0,
$$

where $[D]=[K]^{-1}[M]$ is the dynamic matrix (Çatal 2005), $[I]$ is the unit matrix. The non-trivial solution of (15) is obtained for $\{a\} \neq 0$, thus, the solution of (15) will give the eigenvalues being the frequencies of the system. 
Table 1. Error rates of the FEM frequency values according to TMM.

\begin{tabular}{lccr}
\hline Model With & Mode & MAE & ARE $(\%)$ \\
\hline One & 1 & 9.2 & $0 \cdot 81$ \\
Attached & 2 & 173.62 & 3.62 \\
Mass & 3 & 105.74 & 1.42 \\
Five & 1 & 12.93 & 15.47 \\
Attached & 2 & $78 \cdot 81$ & 17.92 \\
Masses & 3 & 183.74 & 19.69 \\
Ten & 1 & 4.53 & 19.28 \\
Attached & 2 & 29.88 & 19.32 \\
Masses & 3 & 77.95 & 20.33 \\
\hline
\end{tabular}

\section{Numerical analysis}

Natural frequencies for the first three modes of an elastically supported Timoshenko column with 1, 5 and 10 attached masses are computed by both TMM and FEM approaches for parameters of $f=0 \cdot 1-0.25-0.5-0.75-0.99-0.999, \bar{M}_{i}=0 \cdot 1-0.5-1-2 \cdot 5-5-7 \cdot 5-10, \bar{J}_{i}=$ $0 \cdot 1-0.5-1-5-10 . m_{i}=0.32 \mathrm{kNs}^{2} / \mathrm{m}^{2}, L_{i}=1 \mathrm{~m}, E I=1353870 \mathrm{kNm}^{2}, A G=$ $3240000 \mathrm{Kn}, k=2.426, S_{x}=0.00743 \mathrm{~m}^{3}, A=0.04 \mathrm{~m}^{2}, I=0.006447 \mathrm{~m}^{4}$ are the characteristics of the IPB profile column used for the numerical analysis. In FEM the two-node beam elements are used, the attached masses being at those nodes, thus, since each node has two degrees of freedom (dof) the total dof with the support node for the models with five and ten masses are $2(5+1)=12$ and $2(10+1)=22$, respectively, that give close enough results. The model with one mass, however, is subdivided into ten equal beam elements to obtain more sensitive results, thus, the total dof for the entire column is $2(10+1)=22$.

According to (1), the relationship between the connection stiffness $\left(K_{\theta} L / E I\right)$ and the fixity factor $(f)$ is approximately linear when the fixity factor values are between 0.0 and 0.5 and non-linear from 0.5 to unity as shown in figure 5. It can be seen from the graph that as the fixity factor approaches unity the curve increases asymptotically to infinity since the fixity factor of unity is used for theoretically ideal fixed support.

The frequency values of the elastically supported Timoshenko column with 1,5 and 10 attached masses are computed by TMM and FEM. The comparison graphs of the frequency values obtained for the models with 1, 5 and 10 attached masses are presented, respectively, in

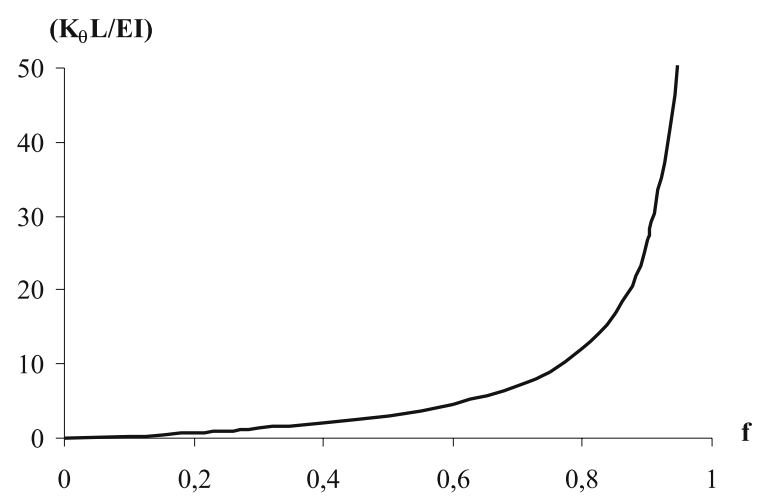

Figure 5. Relationship between the connection stiffness $\left(K_{\theta} L / E I\right)$ and the fixity factor $(f)$. 


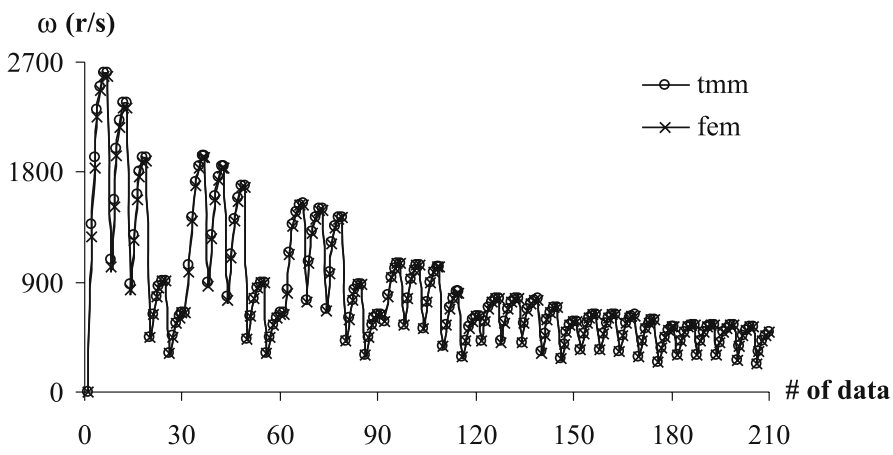

(a) 1.mode
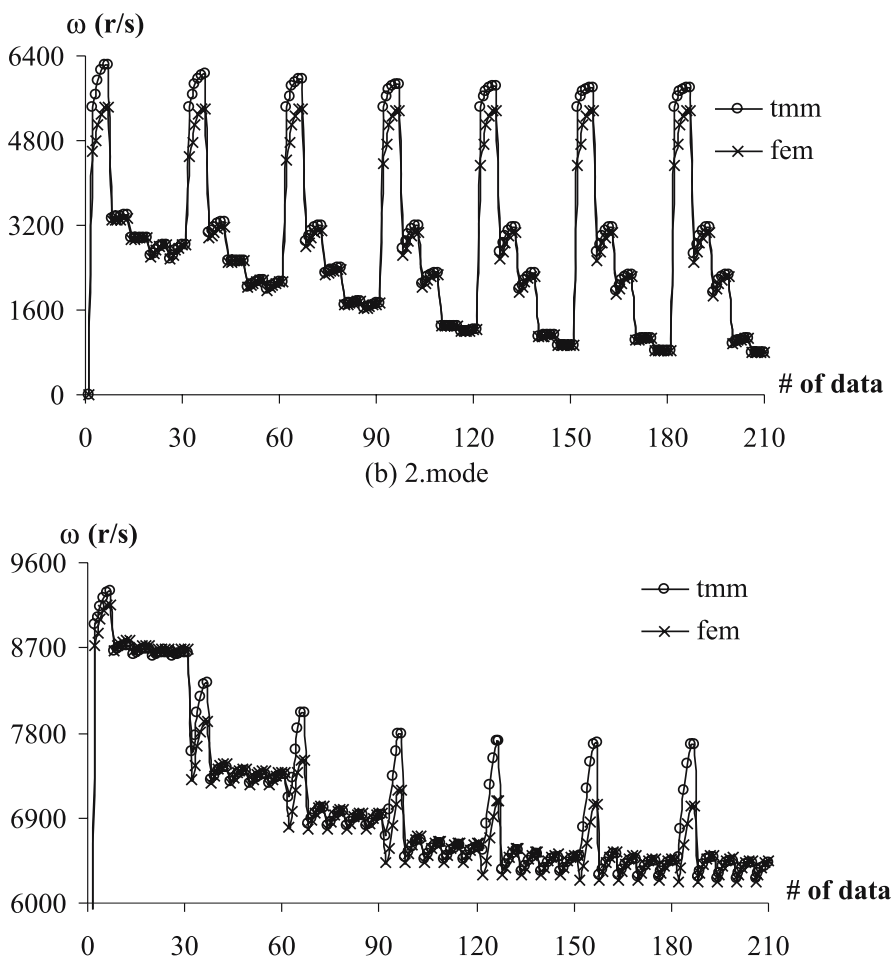

(c) 3.mode

Figure 6. Frequency values of TMM and FEM for the model with 1 attached mass.

figures 6, 7 and 8 for the first, second and third modes. The mean absolute error (MAE $=$ TMM value-FEM value) and average relative error $(\mathrm{ARE}=[\mathrm{MAE} / \mathrm{TMM}$ value $] * 100)$ rates of the FEM models are presented in table 1.

\section{Conclusions}

In this study, elastically-supported Timoshenko column with attached masses is under consideration to obtain its free vibration natural frequencies using two different algorithm; transfer 


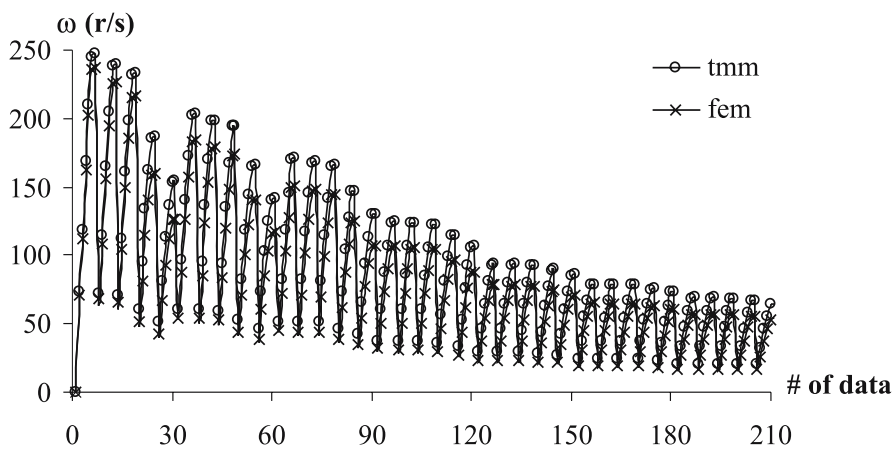

(a) 1.mode

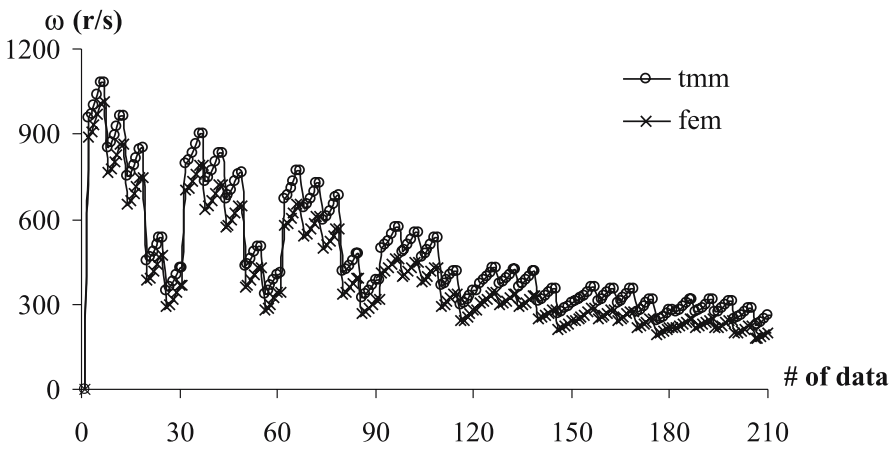

(b) 2. mode

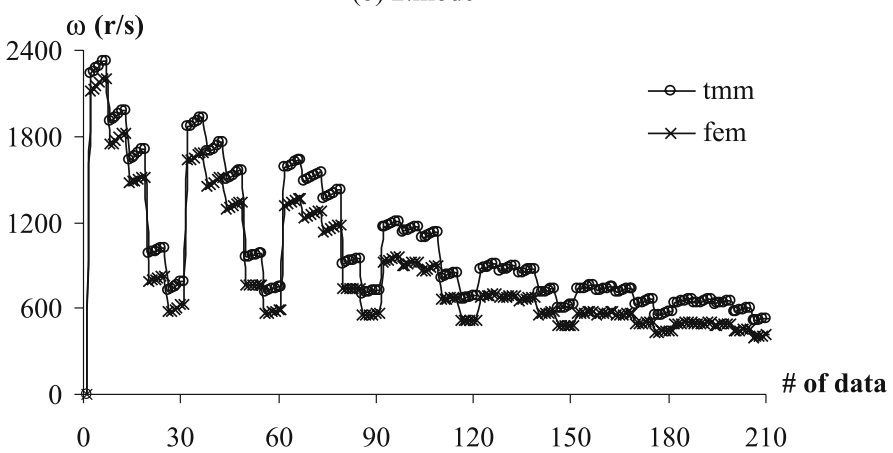

(c) 3.mode

Figure 7. Frequency values of TMM and FEM for the model with 5 attached masses.

matrix method and finite element method. The comparison of the results of two methods is presented in the graphs and the errors of the FEM according to TMM are in a table. According to table 1, FEM gives the best results for the model with one attached mass because of the lowest ARE values since it is subdivided into 10 finite elements; however, the models with 5 and 10 masses are subdivided into 5 and 10 elements, respectively, therefore, ARE values between 15 and $20 \%$ are reached.

For one or two span models it is easy to obtain the frequency equation in explicit form by equating the determinant of coefficient matrix written according to boundary conditions 


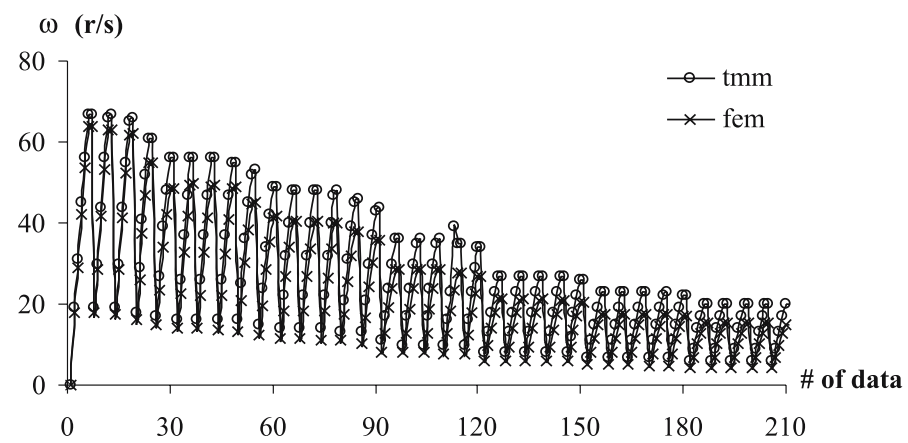

(a) 1.mode

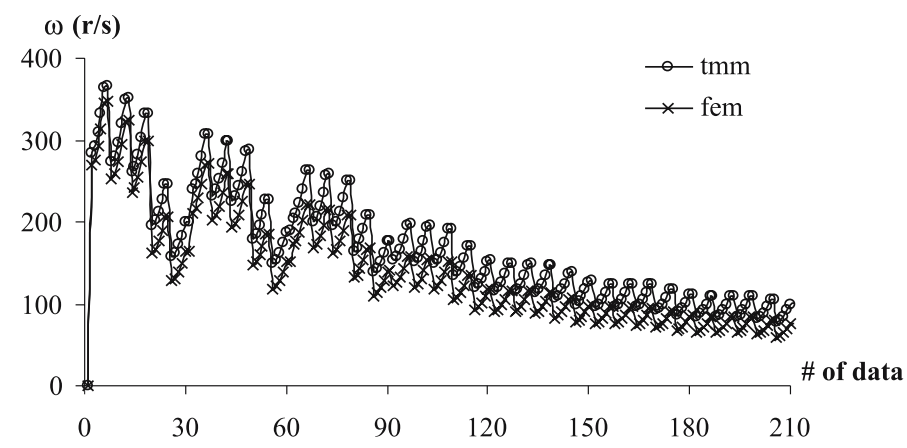

(b) 2.mode

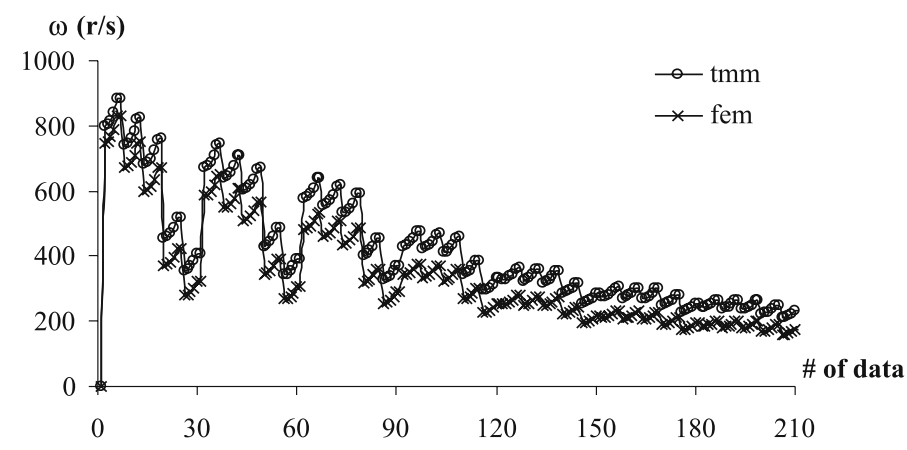

(c) $3 . \operatorname{mode}$

Figure 8. Frequency values of TMM and FEM for the model with 10 attached masses.

of the entire system to zero, however, for large number of spans frequency equation will be extremely complex, therefore, the TMM will be more computationally efficient for these kind of models. In FEM, however, as the number of spans and the attached masses is increased the number of finite beam elements also increases. Thus, the dimension of the matrices formulating the equation of motion will become larger so that the computational effort and time increases. 


\section{References}

Bapat C N, Bapat C 1987 Natural Frequencies of a beam with non-classical boundary conditions and concentrated masses. J. Sound and Vibration 112: 177-182

Cabrero J M, Bayo E 2005 Development of practical design methods for steel structures with semirigid connections. Eng. Struct. 27: 1125-1137

Çatal H H 2005 Matrix methods in structural and earthquake engineering, 2. ed., Turkey, Dokuz Eylül University Engineering Faculty Editions 294: 249-251

Demirdağ O 2005 Obtaining non-linear response spectrum of semi-rigid supported frames, $\mathrm{Ph}$. D. Thesis, Dokuz Eylül University, Izmir, Turkey

Esmailzadeh E, Ohadi A R 2000 Vibration and stability analysis of non-uniform Timoshenko beams under axial and distributed tangential loads. J. Sound and Vibration 236: 443-456

Ferreira A J M, Fasshauer G E 2006 Computation of natural frequencies of shear deformable beams and plates by an RBF-pseudospectral method. Comput. Meth. Appl. Mech. and Eng. 196: 134-146

Gökdağ H, Kopmaz O 2005 Coupled bending and torsional vibration of a beam with in-span and tip attachments. J. Sound and Vibration 287: 591-610

Hurty W C, Rubinstein M F 1964 Dynamics of structures, (India: Prentice Hall) 210-215

Karami G, Malekzadeh P, Shahpari S A 2003 A DQEM for vibration of shear deformable non-uniforms beams with general boundary conditions. Eng. Struct. 25: 1169-1178

Lee U, Kim J, Oh H 2004 Spectral analysis for the transverse vibration of an axially moving Timoshenko beam. J. Sound and Vibration 271: 685-703

Lin H P, Chang S C 2005 Free vibration analysis of multi-span beams with intermediate flexible constraints. J. Sound and Vibration 281: 155-169

Low K H 1991 A comprehensive approach for the eigenproblem of beams with arbitrary boundary conditions. Comput. and Struct. 39: 671-678

Özkaya E 2002 Non-linear transverse vibrations of a simply supported beam carrying concentrated masses. J. Sound and Vibration 257: 413-424

Paz M 1997 Structural dynamics: Theory and computation (USA: Chapmann \& Hall) 405-408

Posiadala B 1997 Free vibrations of uniform Timoshenko beams with attachments. J. Sound and Vibration 204: 359-369

Przemieniecki J S 1968 Theory of matrix structural analysis USA: McGraw-Hill, Inc, 74-80 and 292-297

Rao G V, Saheb K M, Janardhan G R 2006 Fundamental frequency for large amplitude vibrations of uniform Timoshenko beams with central point concentrated mass using coupled displacement field method. J. Sound and Vibration 298: 221-232

Ruta P 2006 The application of Chebyshev polynomials to the solution of the non-prismatic Timoshenko beam vibration problem. J. Sound and Vibration 296: 243-263

Salarieh H, Ghorashi M 2006 Free vibration of Timoshenko beam with finite mass rigid tip load and flexural-torsional coupling. Int. J. Mech. Sci. 48: 763-779

Thomson W T 1981 Theory of vibration with application, 2nd ed., (Englewood Cliffs: USA, PrenticeHall, Inc.) 313-316

Tuma J J, Cheng F Y 1983 Theory and problems of dynamic structural analysis: Schaum's outline series USA: McGraw-Hill, Inc, 157

Wu J J 2006 Use of equivalent mass method for free vibration analysis of a beam carrying multiple two-dof spring-mass systems with inertia effect of the helical springs considered. Int. J. Numer. Meth. Eng. 65: 653-678

Wu J S, Chen D W 2001 Free vibration analysis of a Timoshenko beam carrying multiple spring-mass systems by using the numerical assembly technique. Int. J. Numer. Meth. Eng. 50: 1039-1058

Wu J S, Chiang L K 2004 Free vibration of solid and hollow wedge beams with rectangular or circular cross-sections and carrying any number of point masses. Int. J. Numer. Meth. Eng. 60: 695-718 